\title{
Structural MRI covariance patterns associated with normal aging and neuropsychological functioning ${ }^{\text {th }}$
}

\author{
Adam M. Brickman*, Christian Habeck, Eric Zarahn, Joseph Flynn, Yaakov Stern \\ The Cognitive Neuroscience Division of the Taub Institute for Research on Alzheimer's Disease and the \\ Aging Brain and the Gertrude H. Sergievsky Center, Columbia University Medical Center, New York, NY, USA
}

Received 13 June 2005; received in revised form 10 November 2005; accepted 16 December 2005

Available online 15 February 2006

\begin{abstract}
Structural magnetic resonance imaging (MRI) studies have shown dramatic age-associated changes in grey and white matter volume, but typically use univariate analyses that do not explicitly test the interrelationship among brain regions. The current study used a multivariate approach to identify covariance patterns of grey and white matter tissue density to distinguish older from younger adults. A second aim was to examine whether the expression of the age-associated covariance topographies is related to performance on cognitive tests affected by normal aging. Eighty-four young (mean age $=24.0$ ) and 29 older (mean age $=73.1$ ) participants were scanned with a $1.5 \mathrm{~T}$ MRI machine and assessed with a cognitive battery. Images were spatially normalized and segmented to produce grey and white matter density maps. A multivariate technique, based on the subprofile scaling model, was used to capture sources of between- and within-group variation to produce a linear combination of principal components that represented a "pattern" or "network" that best discriminated between the two age groups. Univariate analyses were also conducted with statistical parametric maps. Grey and white matter covariance patterns were identified that reliably discriminated between the groups with greater than 0.90 sensitivity and specificity. The identified patterns were similar for the univariate and multivariate techniques, and involved widespread regions of the cortex and subcortex. Age and the expression of both patterns were significantly associated with performance on tests of attention, language, memory, and executive functioning. The results suggest that identifiable networks of grey and white matter regions systematically decline with age and that pattern expression is linked to age-related cognitive decline.
\end{abstract}

(C) 2006 Elsevier Inc. All rights reserved.

Keywords: Structural MRI; Aging; Cognition; Multivariate analysis

\section{Introduction}

Voxel based morphometric (VBM) approaches to the analysis of structural magnetic resonance images allow for between- and within-groups comparison of grey and white matter volume or density [6]. Because of its high reliability and semi-automated procedures, VBM is well suited

\footnotetext{
is This research was reviewed and approved by the institutional ethics committee (Institutional Review Board) and written informed consent was obtained from all participants.

* Corresponding author at: Taub Institute for Research on Alzheimer's Disease and the Aging Brain, Columbia University Medical Center, 630 West 168th Street, P\&S 16, New York, NY 20032, USA. Tel.: +1 212342 1348; fax: +1 2123421838 .

E-mail addresses: amb2139@columbia.edu,AMBrickman@gmail.com (A.M. Brickman).
}

for large-scale cross-sectional and longitudinal studies that examine normal age-related neuromorphologic change. In a typical neuromorphologic study of aging, structural magnetic resonance images (MRI) are acquired, spatially normalized to common stereotactic coordinates, and segmented into grey matter, white matter, and cerebrospinal fluid (CSF). Comparisons between groups (i.e., in cross-sectional aging studies) or within groups (i.e., in longitudinal analyses) are made on the segmented images on a voxel-by-voxel basis. Statistical parametric maps (SPM) are generated that reflect differences between or within groups (or the relationship with a continuous variable) in each individual voxel.

Although several studies have used manual and semimanual tracing techniques for the assessment of brain volume change across the adult lifespan (e.g. [28]), relatively few have employed VBM approaches. Those studies that have 
used VBM suggest dramatic normal age-associated changes in both grey and white matter volume. In the largest study to date, Good et al. [21] examined grey matter, white matter, and CSF volumes and concentrations in a group of 465 neurologically healthy adults ranging in age from 18 to 79 years. In addition to regional effects, the authors examined global effects by summing grey matter, white matter, and CSF voxel values and tested linear and non-linear contrasts with age. The authors found a linear decline in global grey matter volume with age and several focal areas of relatively greater ageassociated loss. These regions included the superior parietal gyri, pre- and post-central gyri, insula, cerebellum, anterior cingulate, middle frontal gyrus, Heschl's gyrus, and planum temporale. Though global white matter volume was not significantly associated with age, there were regional effects of age, including bilateral frontal lobe and posterior limbs of the internal capsule.

Other VBM investigations of age-related change have been consistent in demonstrating a tendency for grey matter tissue loss, although they have varied in results regarding white matter and the regional distribution of change. In a longitudinal analysis, Resnick et al. [41] examined grey and white matter change in healthy older adults ranging in age from 59 to 85 and found wide-spread areas of decline for both tissue types. Total grey and white matter volume loss was approximately 5.4 and $2.4 \mathrm{~cm}^{3}$, respectively, with the greatest regional effects in frontal and parietal lobes. Similar to Good and colleagues' findings [21], other cross-sectional analyses of subjects across the adult lifespan revealed a global decline in grey matter density and non-significant age associations for white matter [50,52]. Tisserand et al. [51] reported a significant age-associated decline in grey matter volume with VBM, but they did not evaluate white matter and only analyzed anterior regions.

Although voxel-based approaches to the study of normal aging have generally been consistent with manual tracing or region-of-interest approaches in demonstrating ageassociated grey matter decreases [9,29,36,37], evidence from both human neuroimaging studies and animal studies using other approaches suggests that normal age-related volumetric loss may be primarily due to a decrease in whiter matter $[10,18,24,41]$. Age-associated changes in white matter volume may be reflective of a disturbance in myelin, leading to faulty communication across axons [2]. This change may have particular relevance to cognitive changes associated with normal aging, which may in part be due to decreased communication among critical brain regions. Nonetheless, whether white matter significantly decreases more than grey matter with normal aging has not been demonstrated definitively. Further, the relative impact of grey versus white matter volume on cognitive function requires the comparison of each to cognitive abilities.

Despite the statistical analysis of all of the voxels in the brain, VBM approaches do not consider the interrelationship among voxel densities or volumes. That is, the univariate SPM generated for a VBM analysis provides results for indi- vidual regions and does not address structural connectivity in the brain. For example, although two regionally separate voxels may show aging effects, their relationship to each other is not explicitly examined. In the current study, we employed multivariate spatial covariance methodology to deal with this issue.

An attractive feature of multivariate techniques is their emphasis on structural networks. Because they evaluate the inter-correlation or covariance of tissue density across voxels, the results yielded from the analyses can be interpreted as representational of structural networks throughout the brain. In the current study, we employed the subprofile scaling model (SSM) analytic approach [34] to capture the patterns of age-associated changes in tissue density. This method tests whether, across subjects, tissue densities in individual voxels show a covarying relationship with age as well as amongst each other. Subtle changes in tissue density in the course of aging that also show interregional correlation will be detected by our method, while they might go undetected in a univariate analysis. Different rates of agerelated change at different voxel location are specified by regional weights of both signs (for increasing and decreasing density with age) in a covariance pattern. Identification of such an age-related covariance pattern might be a useful signature of the aging process in the healthy brain. The extent to which each individual subject manifests the age-related topographic pattern can be captured in a single number, similar to a factor score in a factor analysis. The SSM approach has been used in a number of functional neuroimaging studies (e.g. $[11,25,26,43,44,48,49])$, but no study to our knowledge has employed the technique to structural neuroimaging data. Thus, a primary purpose of the current study was to examine whether SSM could identify a covariance pattern of age-related grey and white matter tissue change and to qualitatively compare this approach with a standard univariate VBM analysis of the same dataset.

A secondary aim of the current study was to examine the relationship between the expression of the identified SSM age-associated covariance pattern and performance on a brief battery of neuropsychological tests. Normal ageassociated neurocognitive decline has been well documented (e.g. $[42,45])$. Although no unified theory of normal cognitive aging has emerged from the extant literature, there is a growing consensus that the domains of executive functioning and memory are disproportionately affected $[15,56]$. Despite the growing literature on normal age-associated morphologic changes and the parallel line of research on normal cognitive aging, relatively few studies have investigated the interrelationship among age, neurocognition, and brain morphology (e.g. $[23,38,52,54])$. These efforts have generally demonstrated a relationship between age-associated decline in regional brain volume and performance on tasks of executive functioning and memory, although age typically accounts for more variance in task performance than morphology. In the current study, we sought to identify a grey and white mat- 
ter covariance patterns that captured the effects of age on neuropsychological functioning.

\section{Methods}

\subsection{Subjects}

Data for the current study came from ongoing neuroimaging studies of normal aging. Subject groups included 113 participants, comprising younger $(n=84$, mean age \pm S.D. $=24.02 \pm 3.83$, range $=19-35)$ and older $(n=29$, mean age \pm S.D. $=73.14 \pm 6.72$, range $=60-84)$ individuals. Participants were recruited through local advertisement and word-of-mouth. All subjects were screened with medical, neurological, psychiatric, and neuropsychological evaluations to ensure that they had no neurological or psychiatric disease or cognitive impairment. The screening procedure included a detailed interview that excluded individuals with a self-reported history of major or unstable medical illness, significant neurological history (e.g. epilepsy, brain tumor, stroke), history of head trauma with a loss of consciousness for greater than $5 \mathrm{~min}$, history of diagnosis of an Axis I psychiatric disorder [5]. Individuals taking psychotropic medications were excluded. Older participants were evaluated for dementia with the Mattis Dementia Rating Scale [32] and those scoring below 135 were excluded. Written informed consent, approved by the local ethics committee, was obtained from all participants.

\subsection{Neuropsychological evaluation}

Participants received a brief evaluation of cognitive status. The battery included the Selective Reminding Test (SRT; [17]), the Trailmaking test [39], and the Controlled Oral Word Assocation Test (COWAT; [12]). Overall intellectual functioning was estimated with the New Adult Reading Test (NART), North American version [35]. The SRT is a verbal list learning and memory task in which subjects are given six trials to learn 12 unrelated words. After each trial, the subject attempts to recall the list and is then reminded only of the words that were not recalled. After a 15-min delay, there is a free recall trial followed by a recognition trial, on which words that were not recalled are presented in a multiplechoice array. Measures of total learning, learning on the last trial, long-term retrieval, long-term storage, consistent longterm retrieval, delay free recall, and total recognition are calculated as dependent measures. The Trailmaking test was used a gross measure of attention and executive functioning, other cognitive abilities (i.e., scanning, visuomotor tracking, and motor speed) are required to successfully perform the task [30]. It has two parts. On Part A, the subject is required to connect 25 encircled numbers spread quasi-randomly about a white piece of paper as quickly as possible. On Part B, the subject is required to connect 25 alternating numbers and letters as quickly as possible. The dependent variable for each part is the total time to complete the task. The COWAT is a test of verbal fluency. The subject is given three 60 -s trials to name as many words as possible, beginning with the letters $\mathrm{C}, \mathrm{F}$, and $\mathrm{L}$. The total number of correct words generated is the dependent measure.

\subsection{Image acquisition and analysis}

\subsubsection{Image acquisition}

T1 weighted spoiled gradient (SPGR) images were acquired with a $1.5 \mathrm{~T}$ Philips Intera MRI scanner (TE/TR $=3 \mathrm{~ms} / 34$ $\mathrm{ms}$; flip angle $=45^{\circ} ; \mathrm{FOV}=24 \mathrm{~cm} \times 24 \mathrm{~cm} \times 16 \mathrm{~cm}$; resolution $=256 \times 256 \times 124$ ).

\subsubsection{Image post-processing}

A neuroradiologist reviewed each subject's MRI scan and confirmed that there were no clinically significant findings for any of the subjects. For quantitative investigation, image processing and analysis was conducted with SPM99 (Wellcome Department of Imaging Neuroscience, London) running on Matlab 5.3. Images were first spatially normalized to the same stereotactic space $(7 \times 8 \times 7$ nonlinear basis functions), defined by the Montreal Neurological Institute template. Standard T1 templates of segmented images provided by SPM were used to partition each subject's image into grey matter, white matter, CSF posterior probability images, following standardized procedures [6]. To generate grey and white matter posterior probability images, the images were smoothed with an isotropic Gaussian kernel of $6 \mathrm{~mm}$ fullwidth half-maximum. For statistical analyses, grey and white matter masks were created from the segmented images provided by SPM; these masks set the absolute threshold probability to 0.5 , so that voxels with a probability of being grey or white matter that was less than this cutoff were excluded from the analyses.

\subsection{Subprofile scaling model}

Multivariate analyses were conducted to identify grey and white matter volume correlates of normal aging. The technique is based on SSM, which is a covariance method that has been used in several functional imaging studies on normal aging and neurological disease (e.g. $[25,26,44,48]$ ) and is described in greater detail in an earlier report $[3,33]$. SSM captures the major sources of between- and within-group variation for both the grey and white matter images and produces a series of principal components (PC). For the current study, we sought to identify the optimal number of PCs that should be included as predictors in a linear regression model, using group membership (i.e., younger versus older) as the outcome variable. The best fitting linear combination of these PCs defined an age-related covariance pattern in brain density, capturing most of the variance, while simultaneously yielding the best model fit (explained below). The specific steps of the analysis are as follows: 
1. The re-aligned, spatially transformed and smoothed images from both the elderly and younger participants were simultaneously included in two principal components analysis, one for grey matter images and the other for white matter images. These analyses captured the major sources of between- and within-groups variation and produced a series of principal components. Voxels participating in each PC could have either a positive or negative loading. Positive loadings represent an agerelated increase in volume or density (older $>$ younger), whereas negative loadings represent a decrease in volume or density (older < younger). The loadings were fixed and the same for all subjects. It was predicted that the net loadings would be negative, reflecting greater age-associated tissue loss.

2. The expression of each PC for each subject was quantified by a subject scaling factor (SSF). A higher SSF value indicates greater manifestation of the age-associated PC.

3. To identify a covariance pattern that best discriminates elderly subjects from younger subjects, each individual's expression (SSF) of the specified PCs derived from step 1 was entered into a linear regression model as the predictor variable. Group membership (younger versus older) was the dependent variable. This regression resulted in a linear combination of the PCs that best discriminated between the two groups. The linear combination of the PCs can be thought of as signifying a "pattern" or "network." Akaike's information criterion [16] was used to determine how many PCs should be included in the regression equation in order to achieve optimal bias-variance trade-off. The set of PCs that yielded the lowest value with Akaike's information criterion were selected as predictors in the regression model.

\subsection{Univariate analysis}

Univariate analyses were conducted using standard procedures [6]. Briefly, multiple regression analyses, with age group (i.e., younger versus older) as a covariate, were run separately for grey and white matter images. Statistical parametric maps were created that identified individual voxels in which older adults had significantly reduced grey or white matter density than younger adults. The threshold was set at an uncorrected $\alpha=0.001$. As they fundamentally change the nature of inference [1], neither a global signal covariate nor proportional scaling were used. Further, correction based on the Jacobian determinant was not applied [21]; thus, the spatially transformed images preserved grey and white matter density.

\subsection{Neuropsychological correlates}

Individual subject expressions of grey and white matter covariance patterns, as measured by their SSF, were correlated with performance on each neuropsychological test.
To determine the relationship between the expression of the patterns and cognitive functioning, a series of Pearson product moment correlations was conducted. To examine the relationship between expression of the covariance patterns and cognitive functioning independent of age, partial correlations, controlling for age, were conducted between the expression of the grey and white matter patterns and performance on the neuropsychological tests that evidenced the greatest aging effect. Similarly, to determine the effect of age on cognitive functioning independent of the expression of the grey and white matter patterns, partial correlations, controlling for pattern expression, were conducted between age and cognitive task performance.

\section{Results}

\subsection{Covariance patterns}

Grey and white matter patterns that discriminated between younger and older subjects are displayed in Figs. 1 and 2, respectively. The optimal number of principal components, based on the Akaike criteria [16], was 7 for grey matter and 6 for white matter. Talairach coordinates and their corresponding regional labels for grey matter areas involved in the covariance pattern are displayed in Table 1. Negative factor loadings, indicating collateral age-associated decreases in density, were observed throughout the entire brain, in cortical and subcortical regions. Specific regions included thalamus, cortical and mesial temporal lobe, cortical and subcortical frontal lobe, and parietal lobe (see Fig. 1 and Table 1 for detailed description). As expected, the mean expression of the pattern was significantly greater in the older subjects than in the younger subjects, $t\left(\begin{array}{lll}1 & 1 & 1\end{array}\right)=23.952, p<0.001$. Frequency distributions and a receiver operating curve (ROC), displayed in Figs. 3 and 4, respectively, were derived to determine the cut-point for optimal between-groups discrimination; this analysis demonstrated optimal discrimination between groups with $100 \%$ specificity and $97 \%$ sensitivity.

For white matter, the derived covariance pattern involved negative factor loadings in periventricular regions, including cingulate, corpus callosum, deep frontal lobe white matter, and insula (see Fig. 2 and Table 2 for detailed description). The mean expression of this pattern was significantly greater in older subjects than in younger subjects, $t\left(\begin{array}{lll}1 & 1 & 1\end{array}\right)=19.027$, $p<0.001$, as expected. The frequency distribution and ROC, displayed in Figs. 5 and 6, respectively, indicated an optimal discrimination between groups, with $99 \%$ specificity and 93\% sensitivity.

When examined separately by age group, the relationship between age and expression of the both covariance patterns in the young group, was not statistically significant (both $r$ 's $(84)=0.192, p=0.080$ ). In the older subjects, however, age was significantly associated with both the expression of the grey matter network, $r(29)=0.511, p=0.005$ and 
Table 1

Grey matter areas contributing to the covariance pattern that distinguished between younger and older participants

\begin{tabular}{|c|c|c|c|c|c|}
\hline \multicolumn{3}{|c|}{ Tailarach coordinates } & \multirow[t]{2}{*}{ Hemisphere } & \multirow[t]{2}{*}{ Region } & \multirow[t]{2}{*}{$Z$-value } \\
\hline$X$ & $Y$ & $Z$ & & & \\
\hline 0 & -15 & 6 & Left & Thalamus & 7.04 \\
\hline-38 & -23 & 7 & Left & Superior temporal gyrus & 6.50 \\
\hline 2 & -5 & 9 & Right & Thalamus & 6.48 \\
\hline 55 & -6 & 37 & Right & Precentral gyrus & 6.08 \\
\hline-14 & -33 & 0 & Left & Parahippocampal gyrus & 5.65 \\
\hline 44 & -8 & 4 & Right & Insula & 5.43 \\
\hline-8 & 16 & -1 & Left & Caudate & 5.06 \\
\hline-55 & -10 & 37 & Left & Precentral gyrus & 5.03 \\
\hline-40 & -18 & -1 & Left & Insula & 5.01 \\
\hline 16 & -63 & 25 & Right & Precuneus & 4.96 \\
\hline-50 & 11 & 23 & Left & Inferior frontal gyrus & 4.86 \\
\hline 16 & 10 & 14 & Right & Caudate & 4.79 \\
\hline 46 & -38 & 50 & Right & Inferior parietal lobe & 4.60 \\
\hline 2 & 6 & -5 & Right & Anterior cingulate & 4.47 \\
\hline-2 & 48 & 22 & Left & Medial frontal gyrus & 4.37 \\
\hline-14 & 3 & -12 & Left & Sucallosal gyrus & 4.35 \\
\hline 2 & 29 & 30 & Right & Cingulate gyrus & 4.28 \\
\hline-8 & -65 & 20 & Left & Precuneus & 4.13 \\
\hline 53 & -66 & 2 & Right & Inferior temporal gyrus & 3.97 \\
\hline-2 & -29 & 44 & Left & Paracentral lobule & 3.97 \\
\hline 50 & 11 & 23 & Right & Inferior frontal gyrus & 3.95 \\
\hline 12 & -72 & -5 & Right & Lingual gyrus & 3.81 \\
\hline 51 & 23 & 25 & Right & Middle frontal gyrus & 3.80 \\
\hline-10 & -27 & 44 & Left & Cingulate gyrus & 3.71 \\
\hline-57 & -23 & 42 & Left & Postcentral gyrus & 3.71 \\
\hline-50 & -29 & 44 & Left & Inferior parietal lobe & 3.71 \\
\hline-38 & -1 & 48 & Left & Middle frontal gyrus & 3.67 \\
\hline-65 & -24 & -4 & Left & Middle temporal gyrus & 3.62 \\
\hline 65 & -46 & 12 & Right & Superior temporal gyrus & 3.51 \\
\hline-24 & 33 & 43 & Left & Superior frontal gyrus & 3.47 \\
\hline 8 & -19 & 43 & Right & Paracentral lobule & 3.45 \\
\hline 6 & 2 & 42 & Right & Cingulate gyrus & 3.42 \\
\hline 57 & -19 & 41 & Right & Postcentral gyrus & 3.41 \\
\hline 51 & -23 & 49 & Right & Postcentral gyrus & 3.40 \\
\hline 12 & -79 & 11 & Right & Cuneus & 3.37 \\
\hline-14 & -86 & -13 & Left & Lingual gyrus & 3.29 \\
\hline 16 & 3 & -12 & Right & Subcallosal gyrus & 3.27 \\
\hline 28 & -1 & 52 & Right & Frontal sub-gyral & 3.22 \\
\hline 14 & -56 & 8 & Right & Posterior cingulate & 3.18 \\
\hline 28 & 26 & 48 & Right & Superior frontal gyrus & 3.16 \\
\hline 50 & -50 & -23 & Right & Fusiform gyrus & 3.15 \\
\hline-16 & -66 & 11 & Left & Posterior cingulate & 3.10 \\
\hline
\end{tabular}

Areas are listed reflect negative loadings (i.e., less density in the older group than in the younger group), by degree to which they reliably covary. Z-values represent relative magnitude of pattern expression.

with expression of the white matter network, $r(29)=0.454$, $p=0.013$.

\subsection{Voxel-wise SPM analysis (univariate)}

Results from the univariate SPM analyses revealed grey and white matter differences between younger and older participants. Grey matter reduction (see Fig. 7) was widespread in both anterior and posterior cortical and subcortical regions. White matter volume reduction was most pronounced along the surface of the corpus callosum, as well as in mesancephalic and mesial temporal lobe areas (see Fig. 8). In general, the multivariate and univariate approaches yielded similar regional patterns of age-related differences.

\subsection{Neuropsychological test performance}

As can be seen in Table 3, the younger group performed significantly better than the older group on all measures of the SRT, with the exception of the recognition memory index, and the Trailmaking test Parts A and B. Estimated IQ did not significantly differ between the two groups. Performance on the neuropsychological tasks that differed between younger and older participants was also significantly correlated with age, 
Table 2

White matter areas contributing to the covariance pattern that distinguished between younger and older participants

\begin{tabular}{|c|c|c|c|c|c|}
\hline \multicolumn{3}{|c|}{ Tailarach coordinates } & \multirow[t]{2}{*}{ Hemisphere } & \multirow[t]{2}{*}{ Region } & \multirow[t]{2}{*}{$Z$-value } \\
\hline$X$ & $Y$ & $Z$ & & & \\
\hline-12 & -2 & 28 & Left & Cingulate gyrus & 6.94 \\
\hline 34 & -40 & 11 & Right & Subgyral (temporal lobe) & 6.72 \\
\hline 14 & 4 & 5 & Right & Lentiform nucleus & 5.17 \\
\hline 10 & -6 & -6 & Right & Extra-nuclear & 4.04 \\
\hline-26 & -31 & 2 & Left & Extra-nuclear & 3.68 \\
\hline-30 & -19 & 14 & Left & Insula & 3.67 \\
\hline 32 & -9 & 15 & Right & Insula & 3.64 \\
\hline 26 & -29 & 0 & Right & Extra-nuclear & 3.62 \\
\hline-8 & -8 & -6 & Left & Extra-nuclear & 3.60 \\
\hline 0 & -34 & 20 & Interhemispheric & Corpus callosum & 3.56 \\
\hline 8 & -12 & -4 & Right & Subthalamic nucleus & 3.42 \\
\hline
\end{tabular}

Areas are listed reflect negative loadings (i.e., less density in the older group than in the younger group), by degree to which they reliably covary. $Z$-values represent relative magnitude of pattern expression.

Table 3

Neuropsychological test performance differences between age groups

\begin{tabular}{|c|c|c|c|c|}
\hline Subtest & Young mean (S.D.) & Older mean (S.D.) & $F$ (d.f.) & $p$ \\
\hline SRT total & 61.42 (13.49) & $45.32(12.55)$ & $26.06(1,83)$ & $<0.001$ \\
\hline SRT LTR & $58.59(14.47)$ & $35.56(14.62)$ & $44.25(1,83)$ & $<0.001$ \\
\hline SRT LTS total & $60.19(13.95)$ & $38.56(15.16)$ & $40.07(1,83)$ & $<0.001$ \\
\hline SRT CLTR total & $53.14(16.37)$ & $27.38(15.53)$ & $43.47(1,82)$ & $<0.001$ \\
\hline SRT last trial & $11.27(2.44)$ & $8.84(2.61)$ & $16.72(1,83)$ & $<0.001$ \\
\hline SRT delay free & $10.07(2.62)$ & $7.57(2.89)$ & $14.18(1,80)$ & $<0.001$ \\
\hline SRT recog. & $11.29(2.73)$ & $11.35(2.72)$ & $0.007(1,80)$ & 0.935 \\
\hline NART IQ & $119.82(5.63)$ & 116.95 (8.74) & $3.12(1,80)$ & 0.081 \\
\hline COWAT & $49.00(12.98)$ & $44.12(11.46)$ & $1.74(1,43)$ & 0.195 \\
\hline Trails A & $26.71(11.64)$ & $41.21(17.56)$ & $15.75(1,67)$ & $<0.001$ \\
\hline Trails B & $52.22(22.14)$ & $92.11(31.05)$ & $35.15(1,67)$ & $<0.001$ \\
\hline
\end{tabular}

as expected ( $r$ 's range from 0.417 to 0.640 , all $p$ 's $<0.001$ ). The expression of both grey and white matter covariance patterns was significantly correlated with the learning and free recall measures of the SRT and the Trailmaking test Parts A and B, such that greater expression of the covariance patterns was associated with poorer performance on the neuropsychological tests. The significant correlation coefficients, displayed in Table 4, were of medium to large effect size. Correlation coefficients were of similar magnitude for grey and white matter (all $p$ 's $>0.05$ with Fisher's Z-test).

As significant associations between age, expression of grey and white covariance patterns, and performance on neuropsychological tests were of relatively large magnitude, a more detailed examination of the interrelationship among these variables was conducted. For these analyses, SRT CLTR and Trailmaking test Part B were chosen, as they demonstrated the greatest aging effects, greatest relation to expression of the covariance patterns, and represented two distinct neuropsychological constructs. When the influence of age was removed from scores on the two neuropsychological tests by partialling them out, and correlated with grey covariance pattern expression, the associations with scores on the SRT CLTR remained significant, $r(80)=-0.238, p=0.031$, though the relationship with Trailmaking test Part B did not $r(65)=0.068, p=0.585$. Similarly, the association between expression of the white matter covariance pattern and SRT CLTR remained significant, $r(80)=-0.238, p=0.031$, but its relationship with performance on the Trailmaking test Part B did not, $r(65)=-0.179, p=0.148$. When the influence of

Table 4

Correlation coefficients for the relationship between the expression of grey and white matter covariance patterns and performance on neuropsychological tests

\begin{tabular}{|c|c|c|c|c|}
\hline \multirow[t]{2}{*}{ Subtest } & \multicolumn{2}{|c|}{ White matter } & \multicolumn{2}{|c|}{ Grey matter } \\
\hline & $R$ & $p$ & $R$ & $p$ \\
\hline SRT total & -0.485 & $<0.001$ & -0.530 & $<0.001$ \\
\hline SRT LTR & -0.579 & $<0.001$ & -0.617 & $<0.001$ \\
\hline SRT LTS total & -0.559 & $<0.001$ & -0.603 & $<0.001$ \\
\hline SRT CLTR total & -0.571 & $<0.001$ & -0.618 & $<0.001$ \\
\hline SRT last trial & -0.403 & $<0.001$ & -0.464 & $<0.001$ \\
\hline SRT delay free & -0.390 & $<0.001$ & -0.410 & $<0.001$ \\
\hline SRT recog. & -0.087 & 0.439 & -0.041 & 0.717 \\
\hline NART IQ & -0.149 & 0.186 & -0.119 & 0.291 \\
\hline COWAT & -0.216 & 0.159 & -0.245 & 0.109 \\
\hline Trails A & 0.437 & $<0.001$ & 0.418 & $<0.001$ \\
\hline Trails B & 0.619 & $<0.001$ & 0.577 & $<0.001$ \\
\hline
\end{tabular}



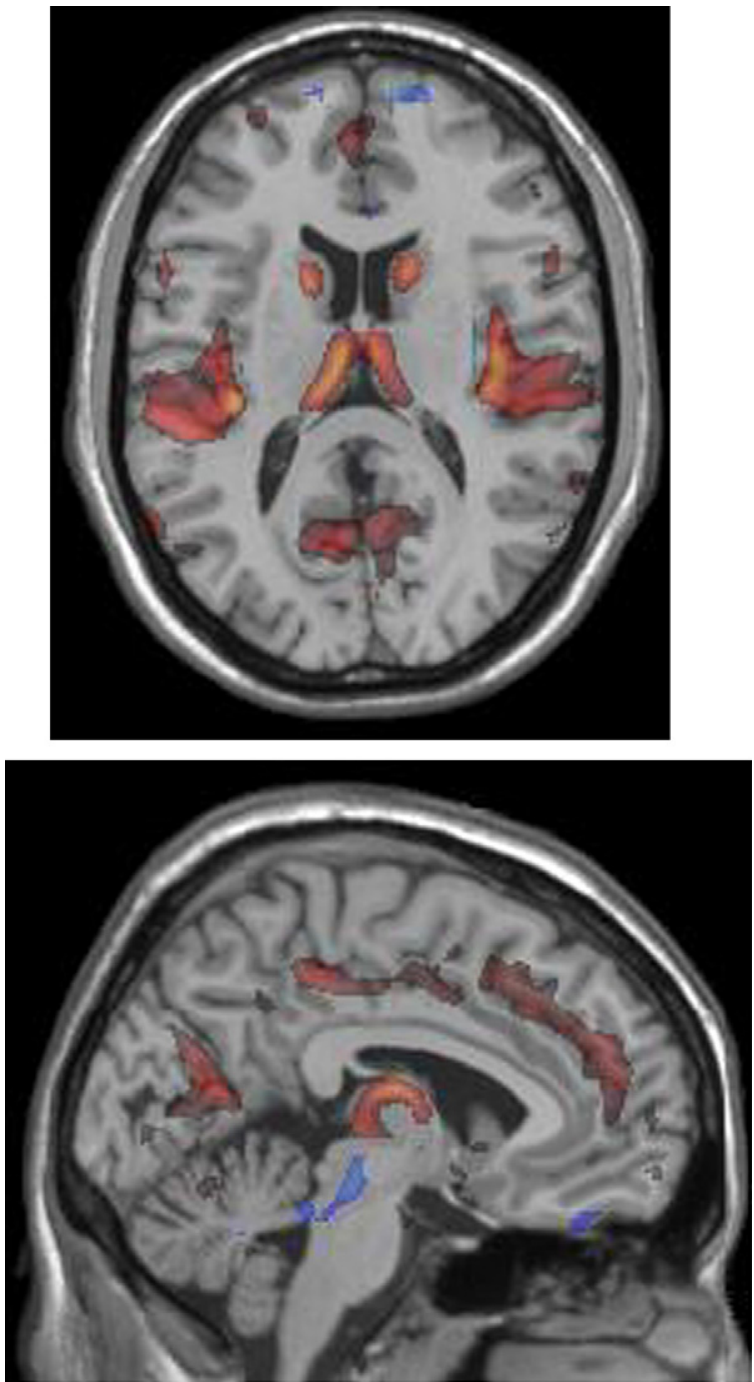

Fig. 1. Axial and sagittal view of grey matter regions implicated in the multivariate covariance SSM analysis. Negative factor loadings, indicating collateral age-associated decreases in density, are displayed in "hot" colors.

the expressions of the grey and white covariance patterns on neuropsychological test performance was removed, associations between age and performance on the SRT CLTR and Trailmaking test Part B remained statistically significant (all $r$ 's $>0.450$, all $p$ 's $<0.001$ ).

\section{Discussion}

In the current study, grey and white matter density was compared between younger and older neurologically healthy participants with a standard univariate VBM approach [6] and a multivariate SSM approach $[33,34]$. Consistent with previous studies of normal age-related changes in neuromorphology, we found age-associated reductions of both tissue types using both analytic techniques. Furthermore, the expression of the identified covariance pattern in each participant was
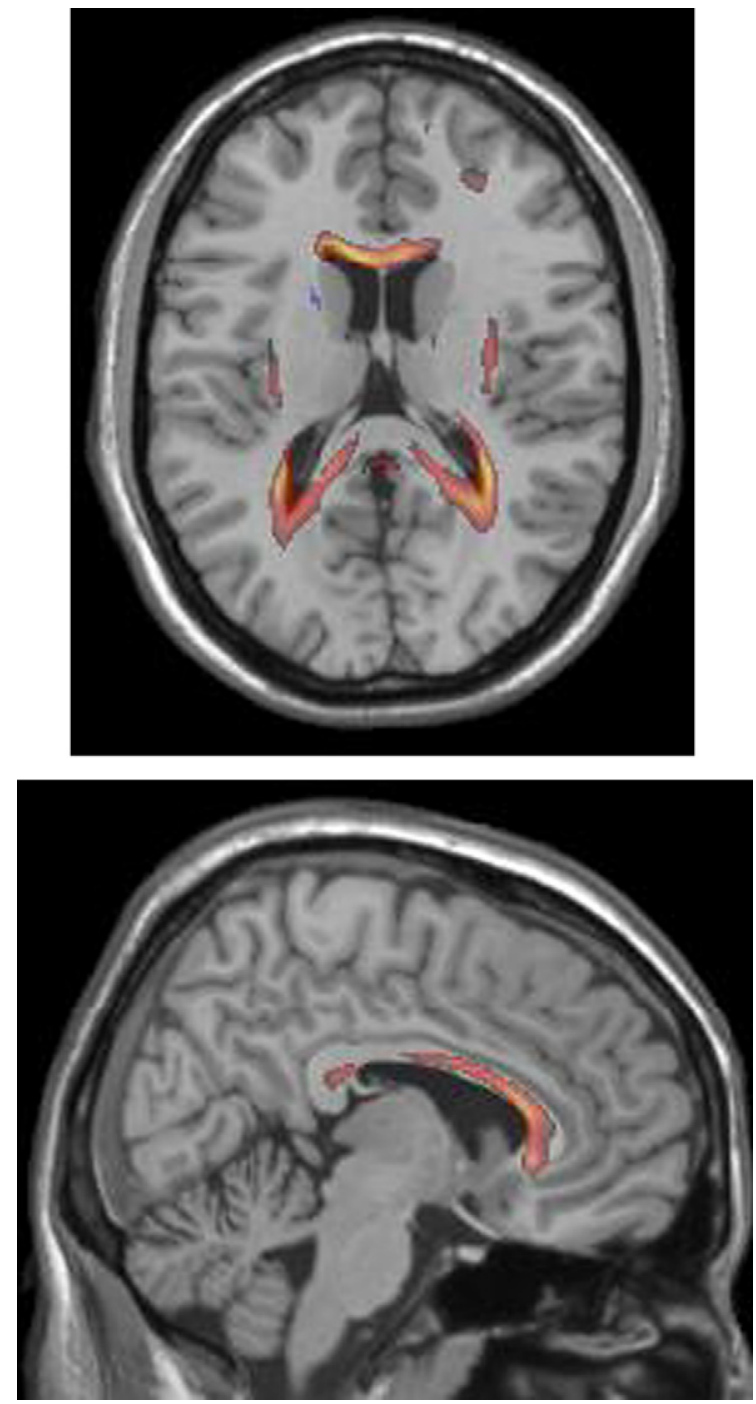

Fig. 2. Axial and sagittal view of white matter regions implicated in the multivariate covariance SSM analysis. Negative factor loadings, indicating collateral age-associated decreases in density, are displayed in "hot" colors.

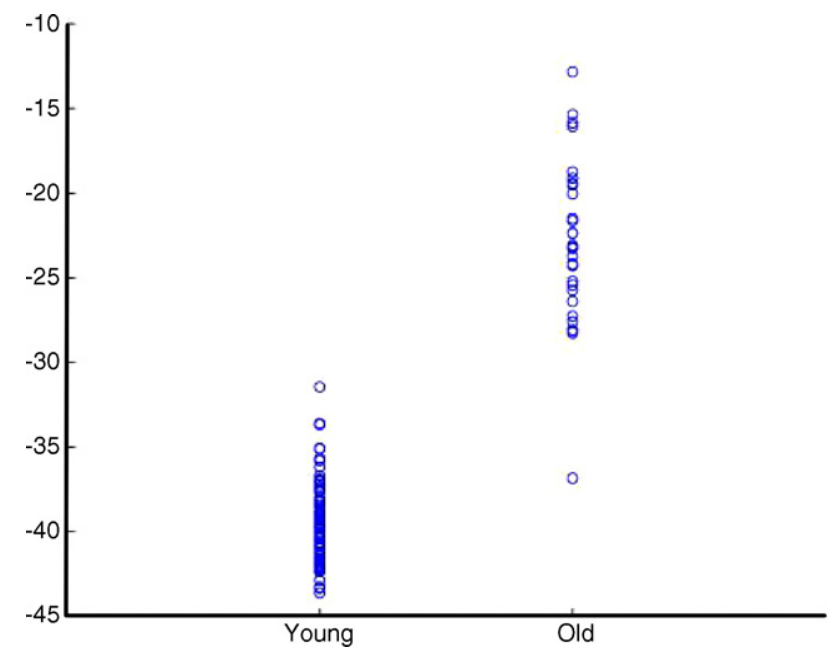

Fig. 3. Distribution of the degree to which younger and older participants expressed the age-associated grey matter topography. 


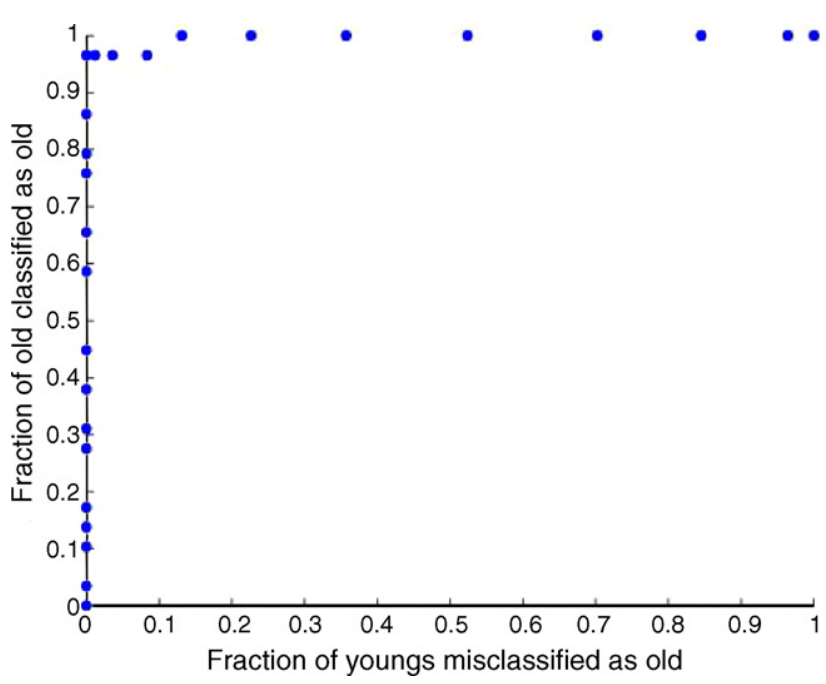

Fig. 4. Specificity and sensitivity plot of the individual expression of grey matter topography.

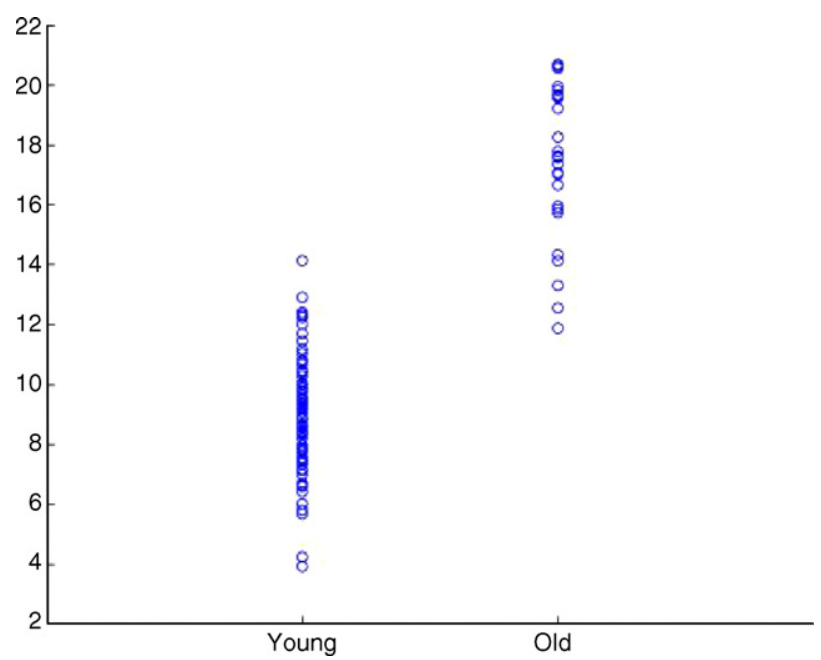

Fig. 5. Distribution of the degree to which younger and older participants expressed the age-associated white matter topography.

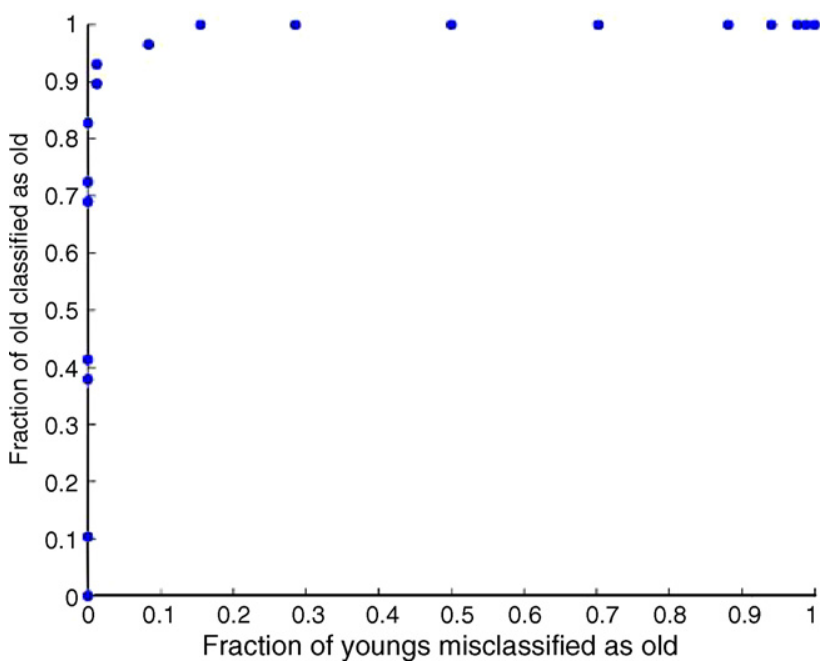

Fig. 6. Specificity and sensitivity plot of the individual expression of white matter topography. significantly related to age, as expected, and to performance on neuropsychological tests of declarative memory, attention, and executive functioning. When controlling for the influence of age, the relationships between the expressions of the covariance patterns and performance on a measure of declarative memory remained statistically significant, though the relationships with performance on a test of executive functioning was reduced to non-significance. When controlling for the expression of grey and white matter covariance patterns, the relationships between age and performance on the neuropsychological tests were reduced, though still statistically significant. These findings suggest that the SSM analysis captured age-associated morphologic changes that partially underlie age-related decline in neuropsychological functioning. Furthermore, they suggest that, independent of age, expression of the identified grey and white matter patterns is related to declarative memory function.

The SSM technique employed in the current study identified interrelated brain regions that best distinguished between young and older groups with greater than $90 \%$ specificity and sensitivity. An advantage of SSM is that it provides a single numeric representation of the expression of the patterns for each individual subject. This parsimonious representation may reflect systematic or uniform morphological changes associated with normal aging. Further, the expression of the grey and white matter patterns was significantly associated with age in the older, but not the younger group, indicating that the relationship between age and morphology is not linear.

The univariate and multivariate approaches identified similar regions that differentiated between younger and older individuals. In terms of the regional distribution, grey matter density differences between the two age groups were widespread and included areas of both cortex and subcortex. Other VBM studies that have examined age-related tissue change have reported similar findings. For example, Resnick et al. [41] found multiple regions of grey matter decline in a longitudinal analysis of elderly neurologically healthy adults. Our findings are also consistent with the largest crosssectional VBM aging study to date, which found an agerelated linear decline in global grey matter volume [21].

The distribution of white matter effects included most of the corpus callosum, deep white matter regions in the frontal and temporal lobe, and some regions in the midbrain. Reports of age-associated changes in white matter have been less consistent. Some studies [8,10,24,40,41], including our own [14], suggest that white matter, particularly in anterior regions, is particularly vulnerable to the effects of normal aging, whereas others have reported relatively little white matter change across the adult lifespan $[21,37]$. Discrepancies among studies could be due, in part, to methodological differences in spatial normalization protocols, sample size differences, tissue segmentation, and the quantification of volume or density. The validity and consistency of tissue segmentation protocols, particularly in older subjects, has not been evaluated well and spatial normaliza- 

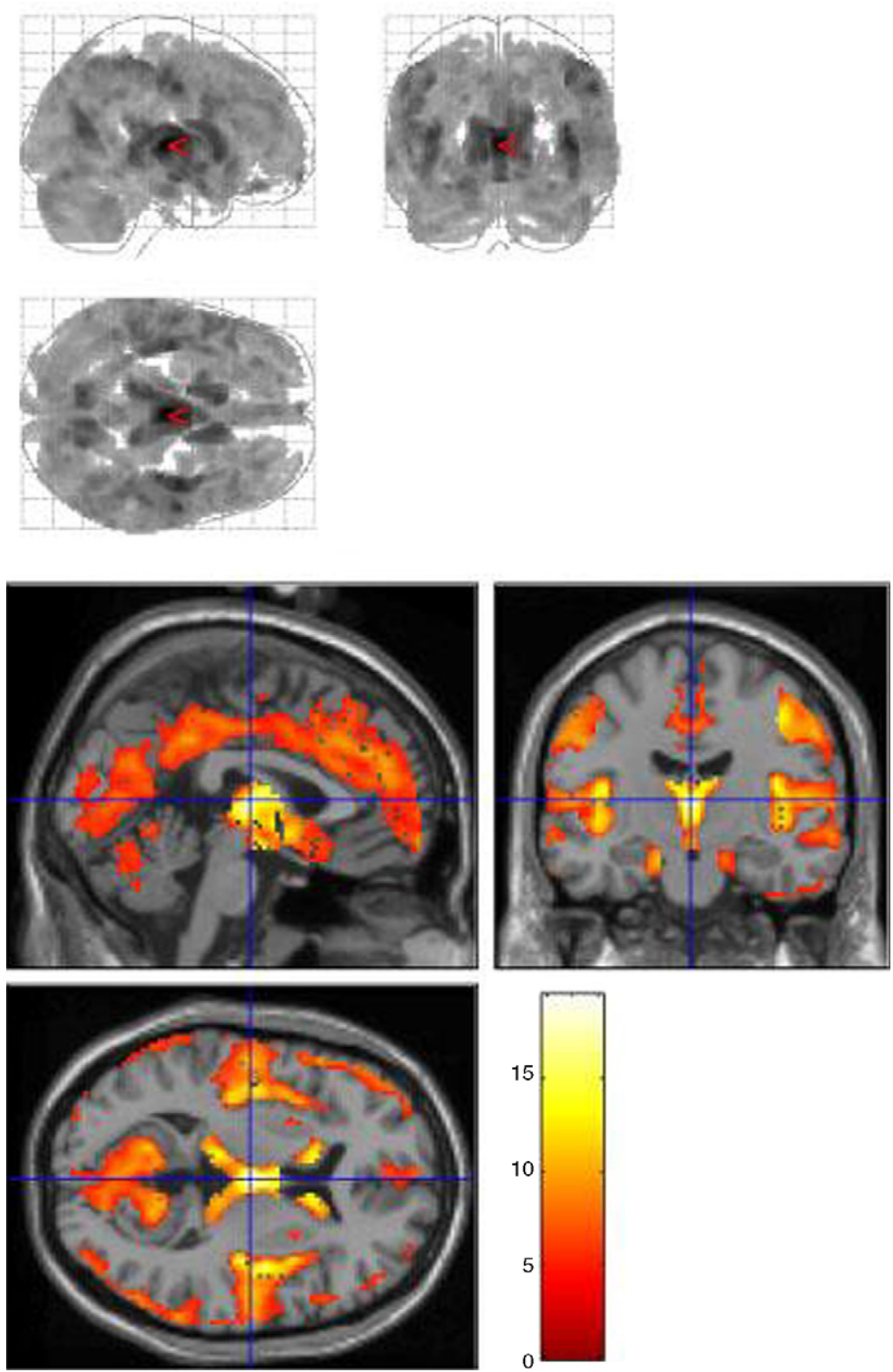

Fig. 7. Statistical parametric maps (SPM) of the standard univariate voxel-based morphometry (VBM) analysis comparing grey matter density between the younger and older groups. The top figure is a "glass brain", which indicates all regions in which older participants had significantly less grey matter density than younger participants, using a grey scale (the voxel with the maximum effect is indicated with the red pointer). The bottom figure shows the same effect superimposed on a standardized brain template. Areas evidencing less grey matter density in older participants compared to younger are indicated with "hot" colors. The color bar represents $t$-values; higher values indicate greater effect sizes.

tion approaches have come under scrutiny for potentially containing systematic biases [13] (cf. [7]). If, for example, there is poorer differentiation between grey and white matter in older subjects, some segmentation protocols may overclassify grey matter and underclassify white matter; this potential error could lead to results suggesting greater white matter than grey matter volume loss with age. Similarly, automated or semi-automated approaches to image analysis may yield complementary, but slightly dissimilar results $[19,22]$ as more traditional region-of-interest volumetry methods, particularly with smaller anatomical structures [51]. Finally, there is recent evidence that age-related changes in white matter volume may not be linear $[4,55]$. Unfortunately, the two-group design of the current study prevented the ability to test for polynomial effects. Thus, although results from the current study suggest that there is both grey and white matter loss with age, they do not speak to the linearity of the effects across the entire adult lifespan. Future studies examining covariance patterns associated with aging should do so in samples that continuously cover the adult lifespan.

Because it captures a pattern of morphological change with a single measurement, the SSM approach used in the 

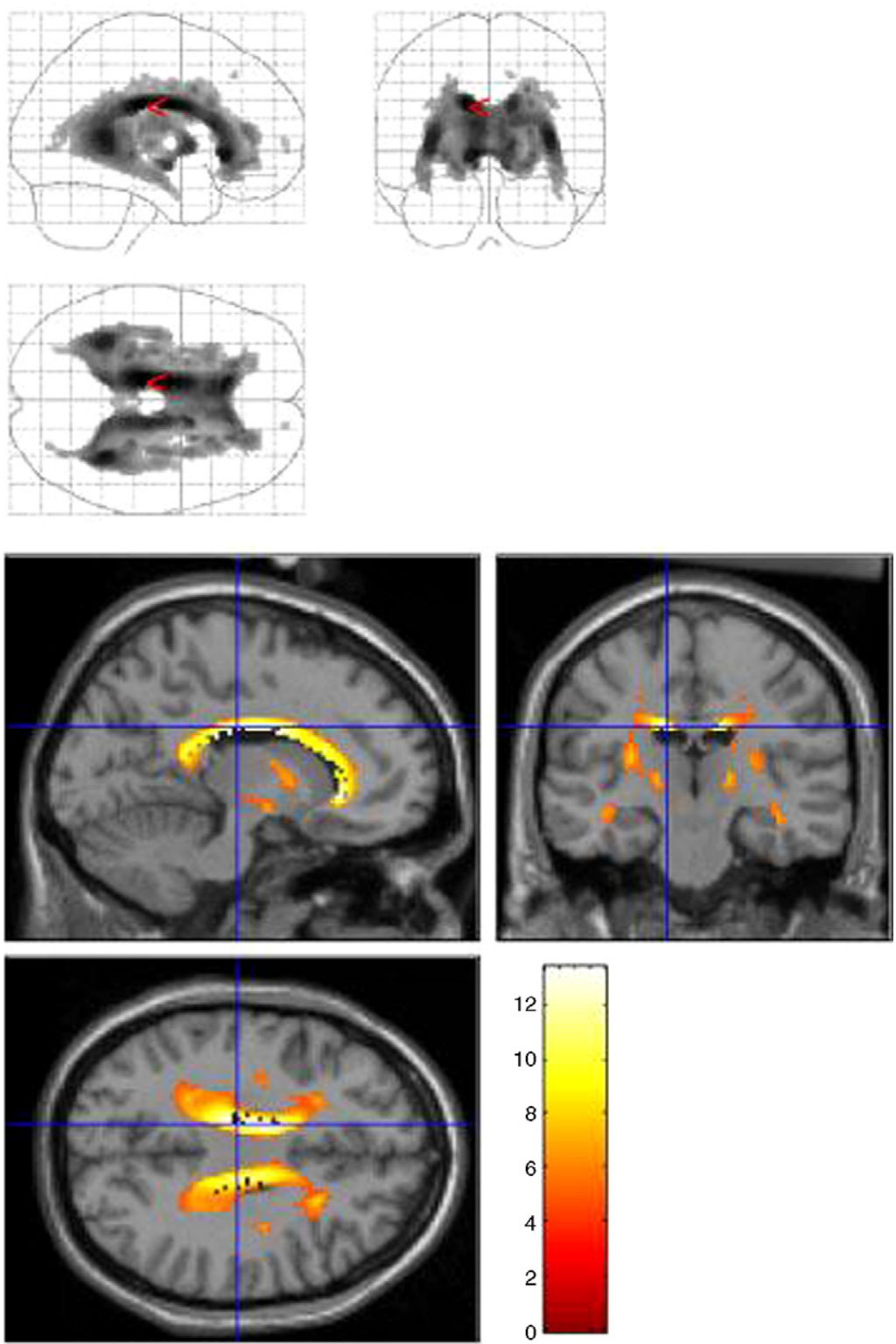

Fig. 8. SPM of the VBM analysis comparing white matter density between the younger and older groups. The top figure is a "glass brain", which indicates all regions in which older participants had significantly less white matter density than younger participants, using a grey scale (the voxel with the maximum effect is indicated with the red pointer). The bottom figure shows the same effect superimposed on a standardized brain template. Areas evidencing less white matter density in older participants compared to younger are indicated with "hot" colors. The color bar represents $t$-values; higher values indicate greater effect sizes.

current study is particularly amenable to the examination of behavioral correlates of age-related structural change. Correlating the degree to which each subject expressed the age-associated topography with performance on tests of neuropsychological functioning partially identified the neuropsychological consequences of age-related structural changes. The correlational analyses suggested that the identified age-related structural networks partially mediated the neuropsychological performance differences between younger and older participants. Other studies have demonstrated significant relationships between the volume of brain regions and performance on neuropsychological tests in older adults $[23,31,38]$. For example, Raz et al. [38] found that a decrease in prefrontal cortex mediated age-related cognitive deficits on a test of executive function, while volume of visual processing areas was associated with performance on tasks of working memory. Examination of subcortical regions has demonstrated associations between reduced thalamic volumes and age-related impairments on tests of cognitive speed [53] and between reduced hippocampal volume and impaired memory performance in the elderly $[20,27]$. The current study sought to determine if the general pattern of age-associated structural brain changes is associated with cognitive changes typically seen in normal aging, as opposed to previous efforts that attempted to identify the specific regions underlying cognitive changes 
with age (e.g. [53]). Thus, direct comparison of the results from the current study to those of past studies that have used a region-of-interest approach or examined a limited number of cerebral regions is difficult. Nonetheless, in the context of past studies that have examined the interrelationship among aging, cognition, and morphology, the current study lends support to the idea that age-related loss of both grey and white matter volume is involved with age-related cognitive change. It should be noted that only a limited battery of tests was used in the current study and thus results might not generalize to other domains of neuropsychological function.

A limitation of the current study was the inclusion of a relatively high intellectually functioning cohort, which may not have been representative of the population. Intellectual functioning may modulate the relationship between structural brain changes and the cognitive manifestation of those changes, as suggested by the theory of cognitive reserve $[46,47]$. However, it was difficult to test this idea in the current study, as the average IQ of the included subjects was quite high and the distribution was constricted. Future research will test the potential moderating role of cognitive reserve explicitly by including a greater number of subjects across a wider range of intellectual functioning. Further, while cognitive reserve may moderate the relationship between brain changes and behavior, other factors may impact the relationship between age and structural variability. Future studies will also seek to determine potential mediating factors of this relationship, which may include historical measures of chronic perfusion secondary to physical activity.

Another potential limitation of the current study is the semi-automatic methodology employed. Both approaches to data analysis required automatic tissue segmentation and spatial normalization of the images to common stereotactic coordinates. Though these methodological features have been applied in several neuroimaging studies, only recently have these techniques been explicitly compared to more traditional region-of-interest volumetry approaches [51]. Voxel-based approaches appear to capture similar information as these other approaches [19]. They may also be more sensitive to group differences because data from individual voxels are considered for analysis, though results may be less accurate for regions with higher degrees of anatomical variability (for discussion, see [51]).

The current study strongly suggests that multivariate covariance techniques, such as SSM, have utility for the analysis of structural MRI data. In addition to yielding similar results as standard univariate analyses, SSM conveniently captures patterns of differences between groups and summarizes the degree to which individual subjects express the anatomical topography. This feature, in particular, allows for the investigation of behavioral correlates of neuromorphology with a high degree of statistical power. Indeed, in the current study, SSM identified grey and white matter topographies that distinguished older and younger neurologically healthy adults, and demonstrated the expression of these topographies was strongly related to age-related changes in neuropsychological functioning.

\section{Acknowledgements}

This work was supported by National Institute on Aging grants AG024708 (AMB) and AG261858 (YS).

\section{References}

[1] Aguirre GK, Zarahn E, D'Esposito M. The inferential impact of global signal covariates in functional neuroimaging analyses. Neuroimage 1998;8:302-6.

[2] Albert M. Neuropsychological and neurophysiological changes in healthy adult humans across the age range. Neurobiol Aging 1993; 14:623-5.

[3] Alexander GE, Mentis MJ, Van Horn JD, Grady CL, Berman KF, Furey ML, et al. Individual differences in PET activation of object perception and attention systems predict face matching accuracy. Neuroreport 1999;10:1965-71.

[4] Allen JS, Bruss J, Brown CK, Damasio H. Normal neuroanatomical variation due to age: the major lobes and a parcellation of the temporal region. Neurobiol Aging 2005;26:1245-60.

[5] American Psychiatric Association. Diagnostic and Statistical Manual of Mental Disorders. 4th ed. Washington, DC: American Psychiatric Press; 1994.

[6] Ashburner J, Friston KJ. Voxel-based morphometry-the methods. Neuroimage 2000;11:805-21.

[7] Ashburner J, Friston KJ. Why voxel-based morphometry should be used. Neuroimage 2001;14:1238-43.

[8] Bartzokis G. Age-related myelin breakdown: a developmental model of cognitive decline and Alzheimer's disease. Neurobiol Aging 2004;25:5-18 [author reply 49-62].

[9] Bartzokis G, Beckson M, Lu PH, Nuechterlein KH, Edwards N, Mintz J. Age-related changes in frontal and temporal lobe volumes in men: a magnetic resonance imaging study. Arch Gen Psychiatr 2001;58:461-5.

[10] Bartzokis G, Cummings JL, Sultzer D, Henderson VW, Nuechterlein $\mathrm{KH}$, Mintz J. White matter structural integrity in healthy aging adults and patients with Alzheimer disease: a magnetic resonance imaging study. Arch Neurol 2003;60:393-8.

[11] Bell-McGinty S, Habeck C, Hilton HJ, Rakitin B, Scarmeas N, Zarahn $\mathrm{E}$, et al. Identification and differential vulnerability of a neural network in sleep deprivation. Cereb Cortex 2004;14:496-502.

[12] Benton AL, Heamsher K, Sivan AB. Multilingual aphasia examination. Iowa City: AJA; 1976.

[13] Bookstein FL. Voxel-based morphometry should not be used with imperfectly registered images. Neuroimage 2001;14:1454-62.

[14] Brickman AM, Zimmerman ME, Paul RH, Grieve SM, Tate DF, Cohen RA, et al. Regional white matter and neuropsychological functioning across the adult lifespan. Biol Psychiatry, in press.

[15] Buckner RL. Memory and executive function in aging and AD: multiple factors that cause decline and reserve factors that compensate. Neuron 2004;44:195-208.

[16] Burnham KP, Anderson D. Model Selection and Multimodel Inference. New York: Springer-Verlag; 2002.

[17] Buschke H, Fuld PA. Evaluating storage, retention, and retrieval in disordered memory and learning. Neurology 1974;24:1019-25.

[18] Franklin RJ, Zhao C, Sim FJ. Ageing and CNS remyelination. Neuroreport 2002;13:923-8.

[19] Giuliani NR, Calhoun VD, Pearlson GD, Francis A, Buchanan RW. Voxel-based morphometry versus region of interest: a comparison of two methods for analyzing gray matter differences in schizophrenia. Schizophr Res 2005;74:135-47. 
[20] Golomb J, Kluger A, de Leon MJ, Ferris SH, Convit A, Mittelman MS, et al. Hippocampal formation size in normal human aging: a correlate of delayed secondary memory performance. Learn Mem 1994;1: $45-54$.

[21] Good CD, Johnsrude IS, Ashburner J, Henson RN, Friston KJ, Frackowiak RS. A voxel-based morphometric study of ageing in 465 normal adult human brains. Neuroimage 2001;14:21-36.

[22] Good CD, Scahill RI, Fox NC, Ashburner J, Friston KJ, Chan D, et al. Automatic differentiation of anatomical patterns in the human brain: validation with studies of degenerative dementias. Neuroimage 2002;17:29-46.

[23] Gunning-Dixon FM, Raz N. Neuroanatomical correlates of selected executive functions in middle-aged and older adults: a prospective MRI study. Neuropsychologia 2003;41:1929-41.

[24] Guttmann CR, Jolesz FA, Kikinis R, Killiany RJ, Moss MB, Sandor T, et al. White matter changes with normal aging. Neurology 1998;50: 972-8.

[25] Habeck C, Hilton HJ, Zarahn E, Flynn J, Moeller J, Stern Y. Relation of cognitive reserve and task performance to expression of regional covariance networks in an event-related fMRI study of nonverbal memory. Neuroimage 2003;20:1723-33.

[26] Habeck C, Rakitin BC, Moeller J, Scarmeas N, Zarahn E, Brown T, et al. An event-related fMRI study of the neural networks underlying the encoding, maintenance, and retrieval phase in a delayed-match-tosample task. Brain Res Cogn Brain Res 2005;23:207-20.

[27] Hackert VH, den Heijer T, Oudkerk M, Koudstaal PJ, Hofman A, Breteler MM. Hippocampal head size associated with verbal memory performance in nondemented elderly. Neuroimage 2002;17:136572.

[28] Jernigan TL, Archibald SL, Fennema-Notestine C, Gamst AC, Stout $\mathrm{JC}$, Bonner J, et al. Effects of age on tissues and regions of the cerebrum and cerebellum. Neurobiol Aging 2001;22:581-94.

[29] Jernigan TL, Press GA, Hesselink JR. Methods for measuring brain morphologic features on magnetic resonance images. Validation and normal aging. Arch Neurol 1990;47:27-32.

[30] Lezak MD, Howieson DB, Loring DW. Neuropsychological Assessment. 4th ed. New York: Oxford University Press; 2004.

[31] MacLullich AM, Ferguson KJ, Deary IJ, Seckl JR, Starr JM, Wardlaw JM. Intracranial capacity and brain volumes are associated with cognition in healthy elderly men. Neurology 2002;59:16974.

[32] Mattis S. Dementia Rating Scale (DRS). Odessa, FL: Psychological Assessment Resources; 1988.

[33] Moeller JR, Ishikawa T, Dhawan V, Spetsieris P, Mandel F, Alexander GE, et al. The metabolic topography of normal aging. J Cereb Blood Flow Metab 1996;16:385-98.

[34] Moeller JR, Strother SC, Sidtis JJ, Rottenberg DA. Scaled subprofile model: a statistical approach to the analysis of functional patterns in positron emission tomographic data. J Cereb Blood Flow Metab 1987;7:649-58.

[35] Nelson HE. National Adult Reading Test. Test Manual. Windsor, UK: NFER-Nelson; 1982.

[36] Pfefferbaum A, Lim KO, Zipursky RB, Mathalon DH, Rosenbloom MJ, Lane B, et al. Brain gray and white matter volume loss accelerates with aging in chronic alcoholics: a quantitative MRI study. Alcohol Clin Exp Res 1992;16:1078-89.

[37] Pfefferbaum A, Mathalon DH, Sullivan EV, Rawles JM, Zipursky RB, Lim KO. A quantitative magnetic resonance imaging study of changes in brain morphology from infancy to late adulthood. Arch Neurol 1994;51:874-87.
[38] Raz N, Gunning-Dixon FM, Head D, Dupuis JH, Acker JD. Neuroanatomical correlates of cognitive aging: evidence from structural magnetic resonance imaging. Neuropsychology 1998;12:95-114.

[39] Reitan RM. Validity of the trail making test as an indication of organic brain damage. Percept Mot Skills 1958;8:271-6.

[40] Resnick SM, Goldszal AF, Davatzikos C, Golski S, Kraut MA, Metter EJ, et al. One-year age changes in MRI brain volumes in older adults. Cereb Cortex 2000;10:464-72.

[41] Resnick SM, Pham DL, Kraut MA, Zonderman AB, Davatzikos C. Longitudinal magnetic resonance imaging studies of older adults: a shrinking brain. J Neurosci 2003;23:3295-301.

[42] Salthouse TA. The processing-speed theory of adult age differences in cognition. Psychol Rev 1996;103:403-28.

[43] Scarmeas N, Habeck C, Anderson KE, Hilton J, Devanand DP, Pelton $\mathrm{GH}$, et al. Altered PET functional brain responses in cognitively intact elderly persons at risk for Alzheimer disease (carriers of the epsilon4 allele). Am J Geriatr Psychiatr 2004;12:596-605.

[44] Scarmeas N, Habeck CG, Zarahn E, Anderson KE, Park A, Hilton $\mathrm{J}$, et al. Covariance PET patterns in early Alzheimer's disease and subjects with cognitive impairment but no dementia: utility in group discrimination and correlations with functional performance. Neuroimage 2004;23:35-45.

[45] Sliwinski MJ, Hofer SM, Hall C. Correlated and coupled cognitive change in older adults with and without preclinical dementia. Psychol Aging 2003; 18:672-83.

[46] Stern Y. What is cognitive reserve? Theory and research application of the reserve concept. J Int Neuropsychol Soc 2002;8:448-60.

[47] Stern Y. The concept of cognitive reserve: a catalyst for research. J Clin Exp Neuropsychol 2003;25:589-93.

[48] Stern Y, Habeck C, Moeller J, Scarmeas N, Anderson KE, Hilton HJ, et al. Brain networks associated with cognitive reserve in healthy young and old adults. Cereb Cortex 2004.

[49] Stern Y, Habeck C, Moeller J, Scarmeas N, Anderson KE, Hilton HJ, et al. Brain networks associated with cognitive reserve in healthy young and old adults. Cereb Cortex 2005;15:394-402.

[50] Taki Y, Goto R, Evans A, Zijdenbos A, Neelin P, Lerch J, et al. Voxelbased morphometry of human brain with age and cerebrovascular risk factors. Neurobiol Aging 2004;25:455-63.

[51] Tisserand DJ, Pruessner JC, Sanz Arigita EJ, van Boxtel MP, Evans AC, Jolles J, et al. Regional frontal cortical volumes decrease differentially in aging: an MRI study to compare volumetric approaches and voxelbased morphometry. Neuroimage 2002;17:657-69.

[52] Tisserand DJ, van Boxtel MP, Pruessner JC, Hofman P, Evans AC, Jolles J. A voxel-based morphometric study to determine individual differences in gray matter density associated with age and cognitive change over time. Cereb Cortex 2004;14:966-73.

[53] Van Der Werf YD, Tisserand DJ, Visser PJ, Hofman PA, Vuurman E, Uylings HB, et al. Thalamic volume predicts performance on tests of cognitive speed and decreases in healthy aging. A magnetic resonance imaging-based volumetric analysis. Brain Res Cogn Brain Res 2001;11:377-85.

[54] Van Petten C, Plante E, Davidson PS, Kuo TY, Bajuscak L, Glisky EL. Memory and executive function in older adults: relationships with temporal and prefrontal gray matter volumes and white matter hyperintensities. Neuropsychologia 2004;42:1313-35.

[55] Walhovd KB, Fjell AM, Reinvang I, Lundervold A, Dale AM, Eilertsen DE, et al. Effects of age on volumes of cortex, white matter and subcortical structures. Neurobiol Aging 2005;26:1261-70.

[56] West RL. An application of prefrontal cortex function theory to cognitive aging. Psychol Bull 1996;120:272-92. 